\title{
Stéréopantographe à palpeur optique pour relevé de surfaces topographiques
}

\author{
par Robert GrRaud, Ingénieur aux Services des Recherches et Essais des Ateliers Neyret-Beylier et Piccard-Pictet
}

\section{Introduction}

A l'occasion d'un essai d'affouillement sur modèle réduit particulièrement important, le service d'Essais et de Recherches des Ateliers Neyret-Beylier et Piccard-Pictite a été amené à concevoir et à réaliser un appareil spécial permettant d'effectuer rapidement le relevé précis des surfaces soumises à l'expérimentation.

Le problème à résoudre se présentait comme suit: le chenal d'expérience, figurant le fleuve, a 12 mètres de longueur sur 5 de largeur; il contient le modèle réduit au $1 / 25^{\text {e }}$ d'un barrage mobile formé de grandes vannes doubles. La présence de l'ouvrage entraìne dans l'écoulement des perturbations qui se traduisent par des modifications de la forme du lit, le fond de celui-ci étant constitué par du sable. Il s'agit, après chaque essai, correspondant à un régime donné du fleuve et à une disposition donnée des vannes, de dresser la carte du lit à une échelle commode. Ce problème s'est déjà posé dans les laboratoires qui s'occupent d'essais de ce genre; mais il s'était en général agi de surfaces plus réduites, donc plus facilement accessibles en tous leurs points. Dans le cas actuel, une solution originale s'imposait étant donné l'échelle particulièrement importante de l'expérience.

\section{Principe de l'Appareil}

L'appareil, dénommé stéréopantographe en raison de la nature des services qu'il est appelé à rendre, permet d'obtenir une carte au $1 / 10^{\mathrm{e}}$, établie par bandes parallèles de $2 \mathrm{~m}$. 50 de longueur, chaque bande correspondant à une surface de $12 \mathrm{mq}$ environ, puisque la largeur est de $5 \mathrm{~m}$.

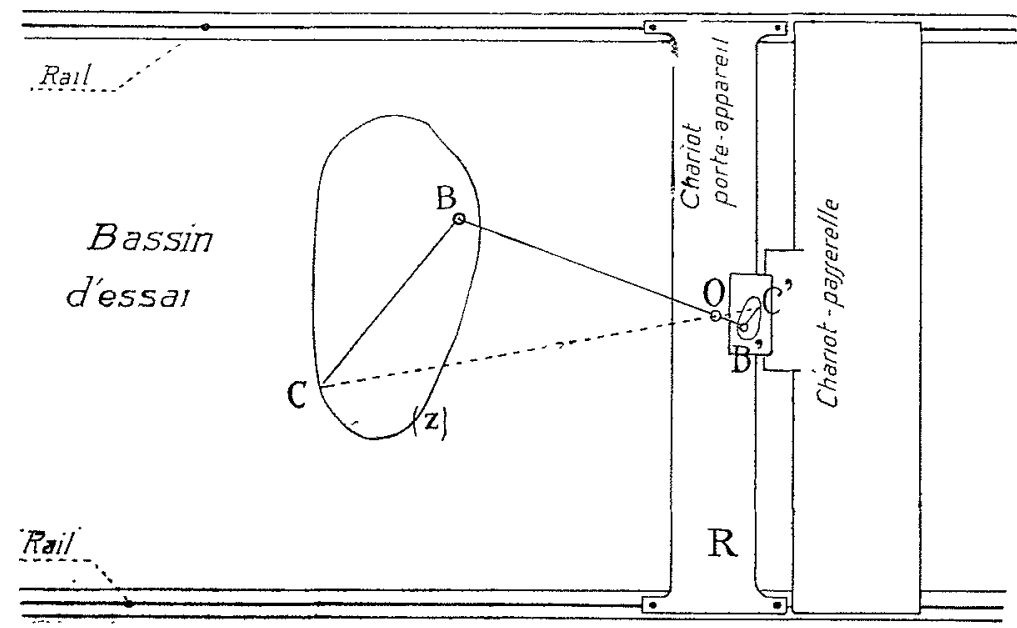

Fig. 1

Le stéréopantographe (fig. 1) est porté par un chariot R, roulant sur des rails au-dessus du chenal à explorer. Il comporte un pivot $O$ autour duquel se déplace un cadre $B O$ B' rigide.
Sur ce cadre est monté un bras explorateur $\mathrm{BC}$, arliculé en $\mathrm{B}$, et un bras traceur B' C', articulé en $B^{\prime}$, ces bras étant constamment parallèles entre eux. On réalise ainsi un système homothétique de centre $O$ et de rapport $1 / 10$.

$$
\frac{1}{10}=\frac{\overline{O B}^{\prime}}{\overline{O B}}=\frac{\overline{B C}^{\prime}}{\overline{\mathrm{BC}}}=\frac{\overrightarrow{O C}}{\overline{O C}}
$$

Enfin en C est fixé un organe permettant de "palper "le terrain à telle cote $(z)$ que l'on veut, cote qui reste invariable durant tout le temps d'une exploration. Grâce à la déformabilité du triangle $\mathrm{O} \mathrm{B} \mathrm{C}$, le point $\mathrm{C}$ peut battre toute la bande de terrain en cours de relevé ; on peut donc déplacer et déformer le triangle $O B C$ de telle sorte que lc palpeur $C$ décrive sur le terrain la courbe de niveau de cote (z) pour laquelle il est réglé ; le point C' décrit alors la courbe homothétique que l'on enregistre facilement sur une feuille de papier en munissant C' d'un organe convenable.

L'originalité principale de l'appareil réside dans le procédé employé pour explorer le terrain. On n'a pas voulu utiliser un palpeur matériel qui, par exemple, aurait pu ètre constitué par une tige pointue suspendue en C. Un tel palpeur est en effet peu commode à deux points de vue : on est obligé de le relever puis de le rabaisser pour franchir chaque ligne de crête et, d'autre part, un contact direct avec le sol conduit à égratigner la surface, ce qui peut en détruire la forme à la moindre incertitude de l'opérateur, pour peu qu'il ait à explorer un endroit délicat. On a, dès lors, eu recours à un palpeur optique imaginé par M. Pierre DaNEL, ingénieur en chef du service des recherches et essais des Ateliers Neyret-Beylier et Piccard-Pictet, palpeur grâce auquel la détermination sur le lerrain des points de mème cote s'effectue sans contact matériel. Le principe de cet appareil it le suivant :

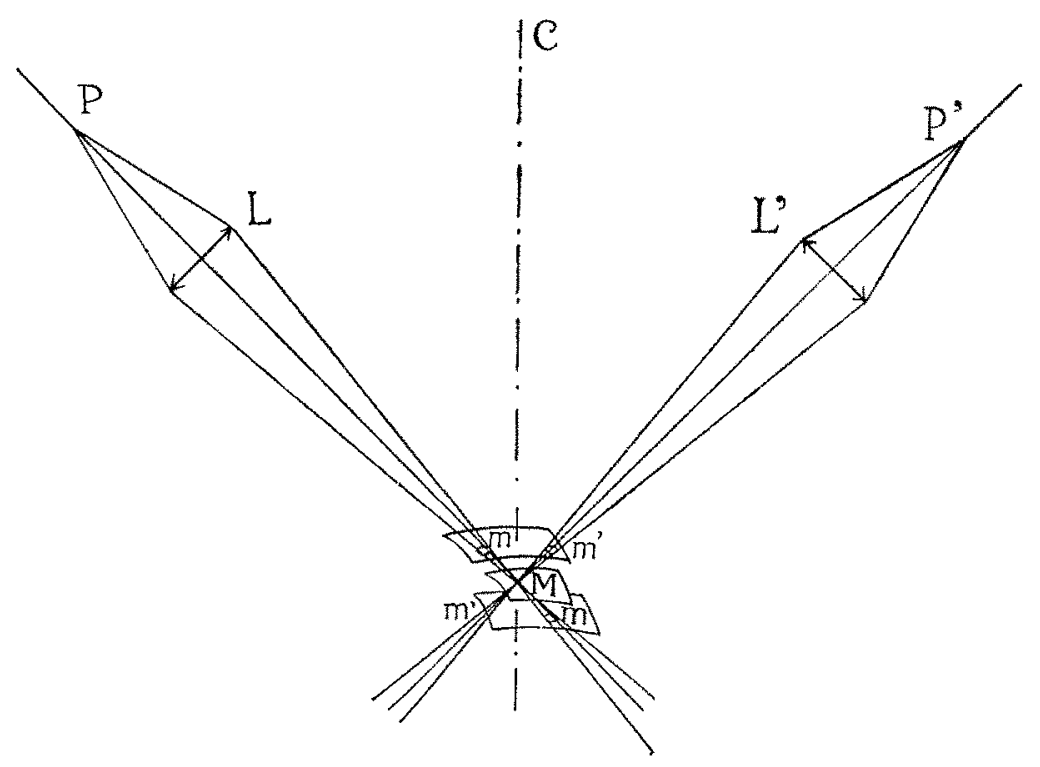

Fig 2 
Deux lentilles, L et L' dont l'axe optique est également incliné sur la verticale du point $C$, donnent de deux points lumineux, $P$ et $\mathrm{P}^{\prime}$, deux images qui, en $\mathrm{M}$, sont confondues sur cette verticale (fig. 2).

L'ensemble du dispositif est fixé sur une tige métallique suspendue en $C$. On a ainsi dans l'espace une image double réelle, $M$, constituée par les deux images confondues dont nous

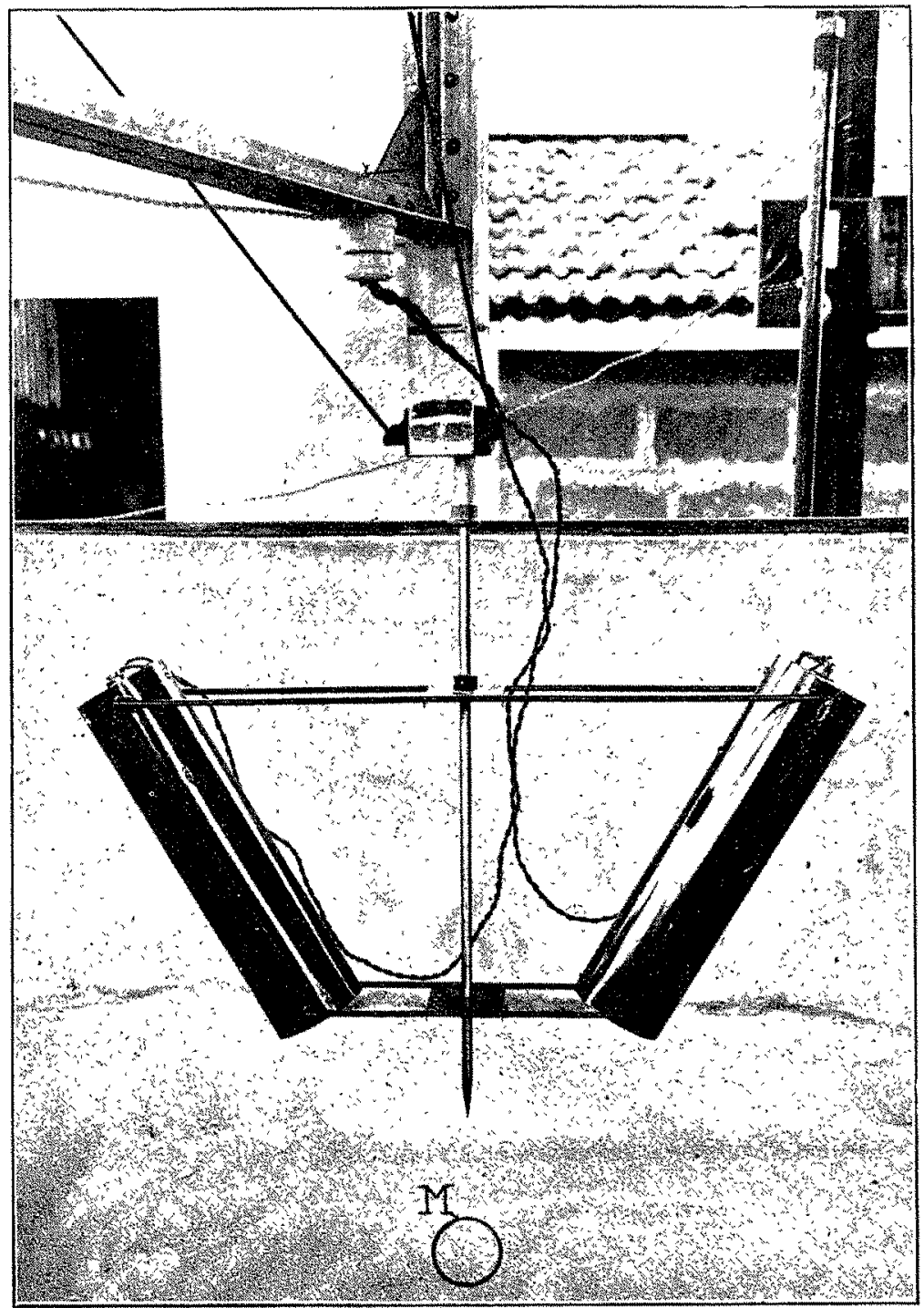

Fig. $3 \div$

venons de parler et qu'il est aisé de régler à une altitude donnée. Pour trouver sur le terrain les points de cote correspondante, il suflit de déplacer $\mathrm{C}$ jusqu'à ce que l'on recoive sur le terrain l'image double $M$; on voil alors une seule tache lumineuse comme sur la photographie (fig. 3). Si l'appareil n'est pas au point, l'aspect change aussitôt et deux taches lumineuses, $m$, m', apparaissent (fig. 4). La précision de la palpation optique est très grande, et plus aisément supérieure à celle de la palpation matérielle dans le cas particulier envisagé. Elle est évidemment fonction de l'angle que font entre eux les axes optiques, de la forme de l'image double $\mathrm{M}$ et de l'acuité visuelle de l'opérateur. Avec le palpeur dont nous donnons la photographie, l'ceil le moins exercé effectue facilement la mise au point de l'image sur le terrain à $3 / 10^{\mathrm{e}} \mathrm{mm}$. près en altitude.

\section{Réalisation pratique. - Réglage et usage de l'appareil}

Procédé de construction. - Les dimensions du stéréopantographe qui permet, complètement développé, d'explorer une zone dont le rayon est de trois mètres, exigeảient une construction à la fois légère et très rigide; légère, pour que l'inertie de l'ensemble ne rende pas malaisée la manœuvre de l'appareil, très rigide pour assurer au maximum, par l'indéformabilité de l'ensemble, la constance et la rigueur des mesures. Aussi a-t-on fait appel au duralumin, profilé en cornière et en plat, la rigidité indispensable étant acquise au moyen de haubans d'acier;

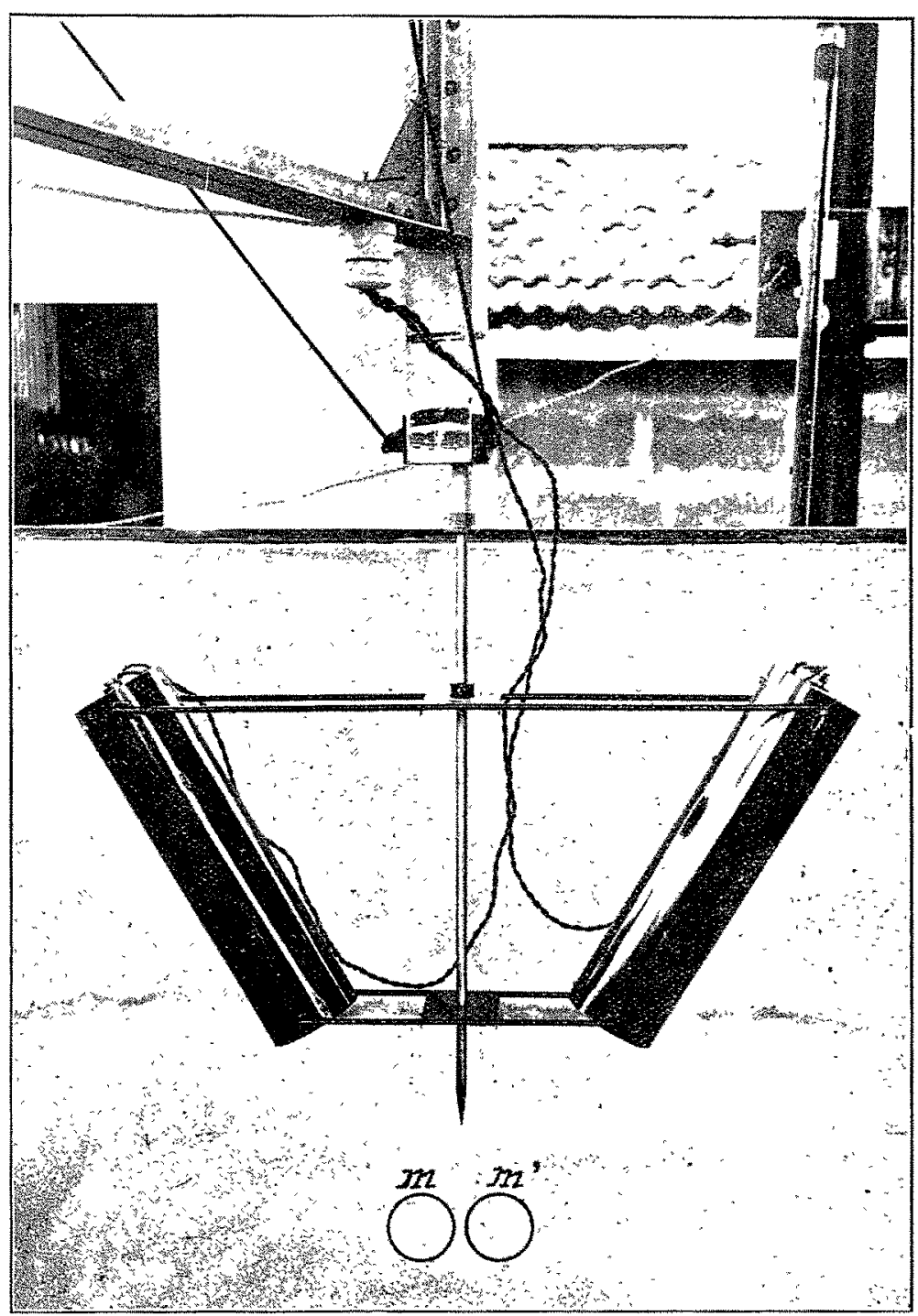

Fig. 4

les assemblages sont faits sur goussets par des rivets de duralumin posés à froid; l'extrémité des haubans est filetée, ce qui permet leur réglage par simple écrou.

Le pivot général $O$ (fig. 1) est constilué par une crapaudine de bronze, montée sur billes, coiffant le sommet d'une colonnette de fonte dont la base porte un chemin de roulement cylindrique où circulent des galets solidaires de la partie inférieure du cadre $\mathrm{B} O \mathrm{O}$ '. Cette disposition rend la rotation fort aisée, tout en soutenant parfaitement l'appareil. La colonnette supportgénéral est boulonnée sur le chariot porte-appareil $R$. Les articulations $B$ et $B^{\prime}$ des bras explorateur et traceur sont montées sur roulement à billes à gorges profondes, leur parallélisme étant obtenu par deux câbles d'acier (convenablement réglés en longueur) enroulés sur des poulies de même diamètre, solidaires de ces bras et centrées sur les arliculations. La photographie (fig. 5) montre que la rotation générale s'effectue par un levier, celle des bras par un volant. L'extrémité du bras traceur C' est munie d'un porte-crayon à ressort ; l'opérateur appuie sur cet organe chaque fois que le palpeur optique est au point; le crayon imprime alors sa trace sur une feville de 
papier portée par une planchette à dessin fixce au support général.

Les figures 3 et 4 montrent suffisamment la structure très simple du palpeur optique; nous n'y insisterons point. Le stéréopantographe est muni d'un dispositif de poulies el de câbles (visibles à mi-hauteur de l'appareil sur la photographie de la fig. 5) grâce auquel le palpeur ou tout autre organe suspendu en C garde toujours la même orientation quelle que soit sa position au-dessus du bassin. Tout l'ensemble est soigneusement équilibré, grâce à des flèches qui portent des contrepoids des pelits colés du chariol; le troisième est constitué par un lube dont chaque extrémité porle une fiole de verre où unc pointe recourbée permet de relever au $1 / 20 \mathrm{~mm}$. le niveau du liquide qui le remplit loul entier el permel ainsi de caler horizontalement les grands cólés de ce même chariol. Cel aménagement permet donc, à chaque station, d'amener le stéréopanlographe rigoureusement dans la même posilion, el de conserver, par conséquent, le bénéfice du réglage initial. Ce dernier a élé effectué en remplissant le bassin d'essais avec de l'eau, ce qui réalise un plan rigourcusement horizontal auquel

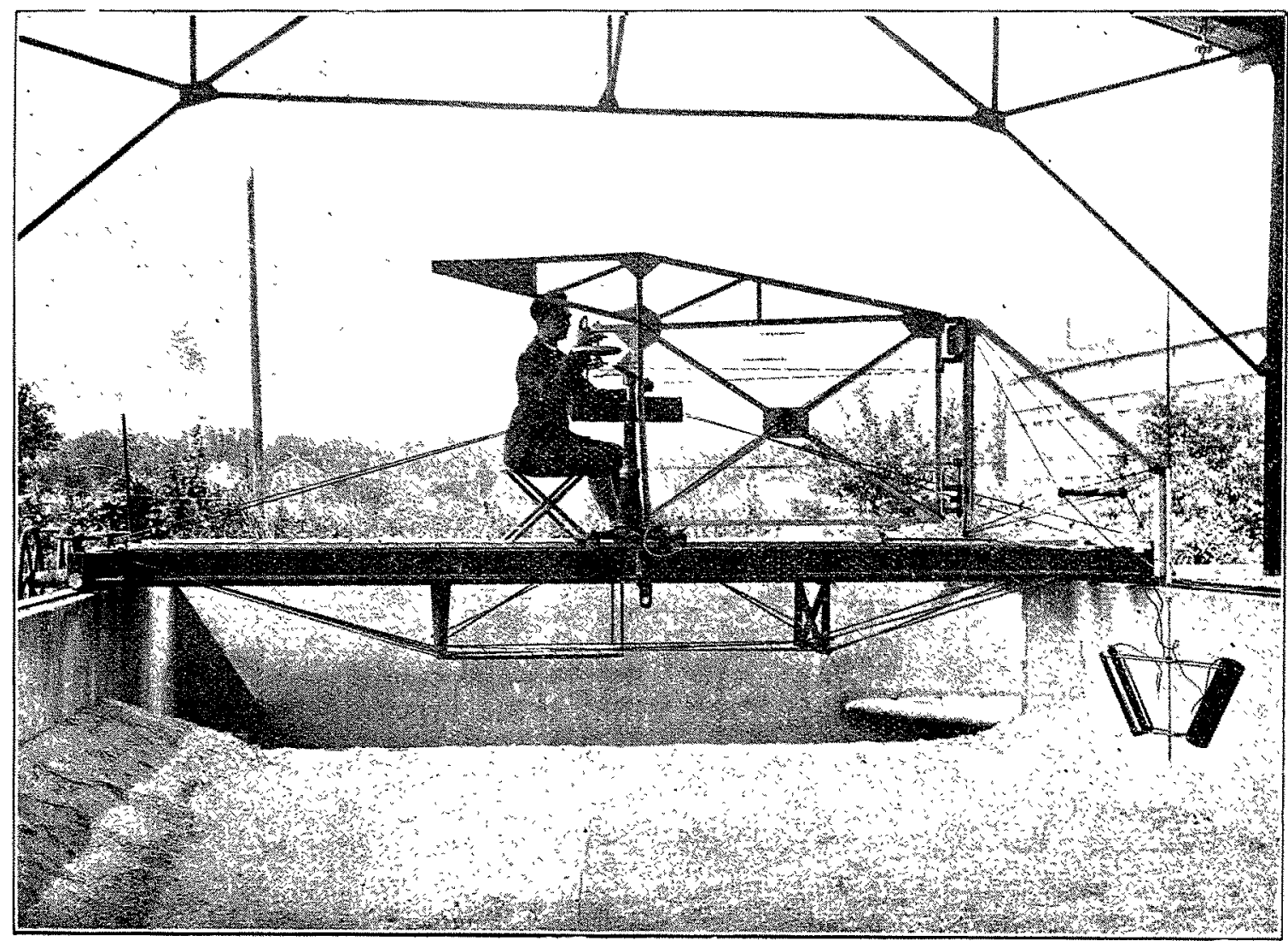

Fig. 5

convenables. Le support général n'est donc soumis à aucun effort de flexion; en outre, le déplacement des différents organes en est facilité.

Le chariot porte-appareil a élé l'objel d'un soin particulier ; il importe en effet que le point lumineux du palpeur se neuve rigoureusement dans un plan horizontal, ce qui implique une rigidité très grande du chariot; elle a été acquise au moyen de haubans d'acier disposés pour combattre à la fois les flexions et les torsions. Toute flèche parasite due à l'opérateur a élé évitée par la construction d'un chariot passerelle indépendant d'où l'on manceuvre l'appareil. Le chariot-passerelle et le chariot porte-appareil sont munis de galets de ronlement; ils se déplacent très facilement à la main et ont chacun un volantfrein.

\section{Réglage de l'Appareil on usage.}

L'appareil a été réglé de la manière suivanle : le chariot porte-appareil comporte 4 vis calantes et 3 niveaux d'caux; 2 d'entre eux, de forme ordinaire, à bulle, règlent l'horizontalité on se réfère pour voir si le palpeur se déplace conslamment à la même altitude quand on manoeuvre l'appareil. Il est à peine besoin de souligner la très grande précision que l'on atleint dans le réglage par ce procédé. L'erreur commise dans l'usage du stéréopantographe peut être estimée à quelques dixièmes de $\mathrm{mm}$. au maximum, et elle provient principalement de ce que la mise en station peut n'ctre pas absolument correcte et conforme à la position de réglage. La vérification de l'appareil est d'ailleurs très facile : on n'a qu'à mettre l'appareil en station el à se rapporter de nouveau à un plan d'eau dans le bassin.

L'usage de l'appareil s'esl révélé très commode et rapide. Un opérateur exercé arrive à suivre les lignes de niveau sur le terrain à relever, avec assez de facilité pour que les temps morts de l'exploration soient très réduits. Pratiquement, un relevé du terrain du hassin d'essai ne demande que quelques heures.

En résumé, le stéréopantographe réalise un appareil très précis permettant le relcvé commode de surfaces lopographiques d'une assez grande élenduc. Il est appelé à rendre les plus grands services dans les études d'affouillements sur modèles réduits. 